\title{
A Simple Parity-Time-Symmetric Optoelectronic Oscillator Based on All-Fiber Coupling
}

\author{
Ege Özgün ${ }^{1, *}$, Faruk Uyar ${ }^{1}$, Tolga Kartaloglu ${ }^{1}$, Ekmel Ozbay ${ }^{1,2}$, and Ibrahim Ozdur ${ }^{3}$ \\ ${ }^{1}$ NANOTAM-Nanotechnology Research Center, Bilkent University, 06800 Ankara, Turkey \\ 2 Department of Physics, Department of Electrical and Electronics Engineering and UNAM-Institute of \\ Materials Science and Nanotechnology, Bilkent University, 06800 Ankara, Turkey \\ ${ }^{3}$ Electrical and Electronics Engineering Department, TOBB University of Economics and Technology, 06560 \\ Ankara, Turkey \\ *Author e-mail address: ozgune@bilkent.edu.tr
}

\begin{abstract}
We experimentally demonstrate a simple parity-time-symmetric optoelectronic oscillator based on all-fiber coupling with single-mode microwave oscillation at $9.5 \mathrm{GHz}$ possessing a phase noise value of $-116.2 \mathrm{dBc} / \mathrm{Hz}$ at $10 \mathrm{kHz}$ offset. $\odot 2021$ The Author(s)
\end{abstract}

\section{Introduction and Experimental Setup}

Optoelectronic oscillators (OEOs) are platforms for creating single-mode low phase-noise microwave signals [1]. Despite their advantages, their performance is limited with the quality factor of the RF filter used in the setup. To overcome this problem, various schemes, including dual loop [2,3] and high-Q optical filter [4,5] were proposed.

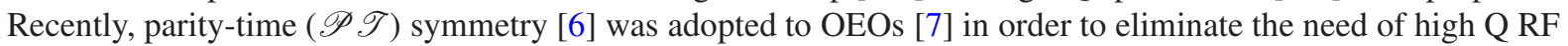
filters.

In this paper, we experimentally demonstrate a simple $\mathscr{P} \mathscr{T}$-OEO based on all-fiber coupling only. The demonstrated setup generates single-mode microwave oscillations at $9.5 \mathrm{GHz}$ with a phase noise value of $-116.2 \mathrm{dBc} / \mathrm{Hz}$ at $10 \mathrm{kHz}$.

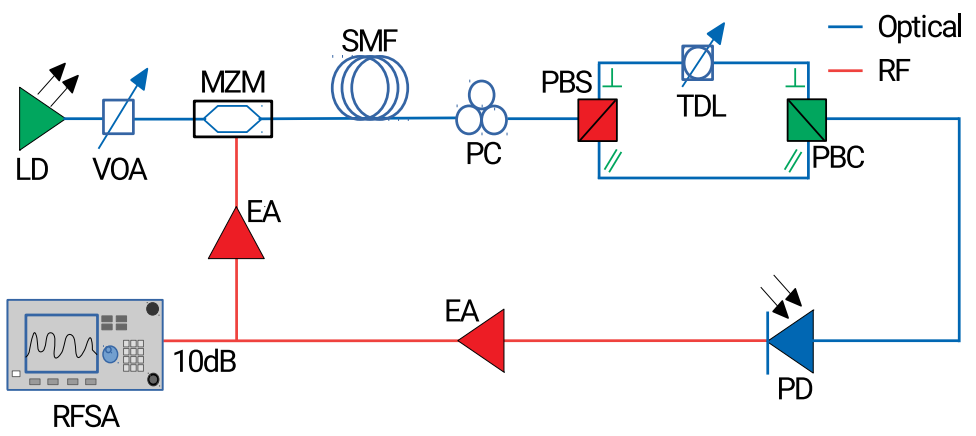

Fig. 1: Block diagram of the experimental setup. LD: Laser Diode, VOA: Variable Optical Attenuator, MZM: Mach-Zehnder Modulator, SMF: Single Mode Fiber, PC: Polarization Controller, PBS: Polarization Beam Splitter, TDL: Tunable Delay Line, PBC: Polarization Beam Combiner, PD: Photodetector, EA: Electrical Amplifier, RFSA: Radio Frequency Spectrum Analyzer.

The coupled mode formalism for a two loop-coupled configuration yields the following eigenmodes [7]

$$
\Omega_{n}^{(1,2)}=\omega_{n} \pm \sqrt{\kappa_{n}^{2}-\gamma_{n}^{2}}
$$

where, $\Omega_{n}^{(1,2)}$ are the eigenfrequencies of the system, $\omega_{n}$ is the frequency prior to coupling, $\kappa_{n}$ is the coupling coefficient and $\gamma_{n}$ is the gain/loss amplitude $\left(g_{n} / l_{n}\right)$, which are equal for the $\mathscr{P} \mathscr{T}$-symmetric configuration and $n$ is the mode index. Above eigenspectrum is neutral, i.e. not oscillating when $\kappa_{n}>\gamma_{n}$. When for any mode $n, \gamma_{n}$ becomes larger than the coupling coefficient, single-mode oscillation for that mode onsets.

The experimental setup, shown in Fig. 1, consists of optical and RF sections. A coherent light source is fed into to the Mach-Zehnder modulator after passing through a variable optical attenuator. Then it passes through a $1 \mathrm{~km}$ length single-mode optical delay line and separated into two arms via a polarization beam splitter. The power balance in two arms is controlled via a polarization controller. To balance the RF phases in two arms, a tunable delay line is implemented into the upper arm. Two arms are then connected by a polarization beam combiner. All the components in two arms are polarization maintaining, including the tunable delay line. The combined signal is detected via the photodetector. Due to the orthogonal polarizations no light interference effect is observed after photodetection. The RF-converted signal is then electrically amplified and separated into two via a $10 \mathrm{~dB}$ coupler. One arm is fed back into the modulator to complete the loop and the other arm is used for measurements, through the RF spectrum analyzer. 

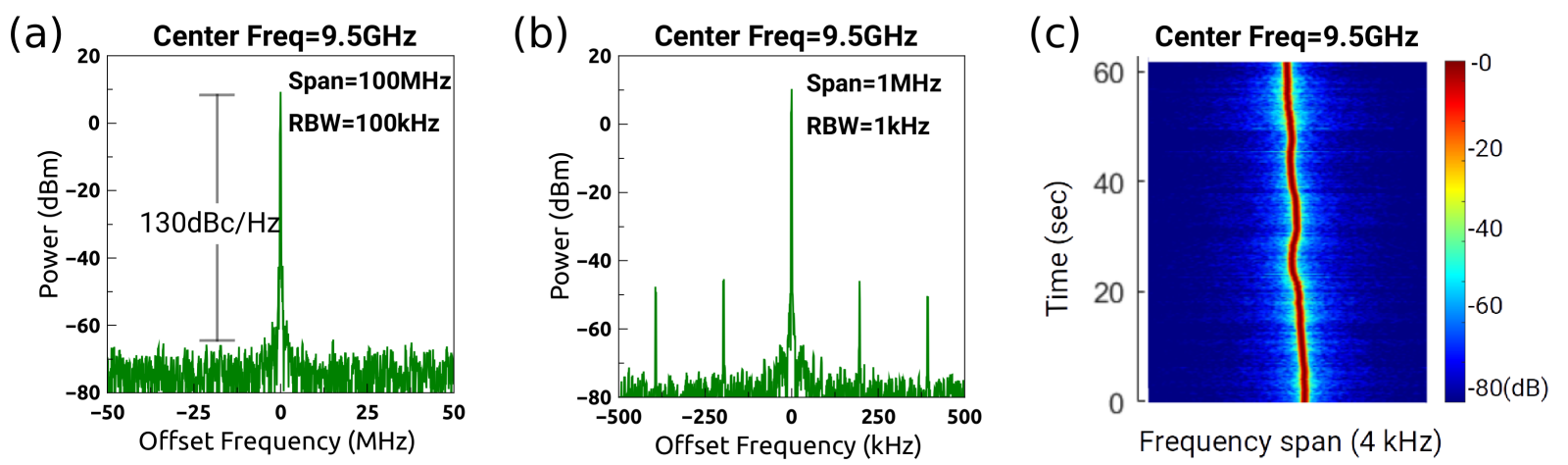

Fig. 2: (a) and (b) RF spectrum traces with different spans. (c) Spectogram over 60 seconds.

\section{Results}

When $\mathscr{P} \mathscr{T}$-symmetry is maintained by balancing gain and loss amplitudes, and then it is broken by satisfying the condition $\kappa_{n}<\gamma_{n}$, single-mode microwave oscillation is obtained. Fig. 2.a shows the oscillating mode at $9.5 \mathrm{GHz}$ together with noise spectral density, for a span of $100 \mathrm{MHz}$ with a resolution bandwidth of $100 \mathrm{kHz}$. Fig. 2.b displays the RF spectrum trace at the same frequency for a span of $1 \mathrm{MHz}$ and a resolution bandwidth of $1 \mathrm{kHz}$. The supermode noise spurs can be seen at $\sim 200 \mathrm{kHz}$ and its harmonics. The spectogram data for one-minute duration is given in Fig. 2.c. The frequency stability of the $\mathscr{P} \mathscr{T}$-OEO is measured to be better than $200 \mathrm{~Hz}$ over 60 seconds. Finally, phase noise is measured and shown in Fig. 3. The phase noise graph starts at $-65 \mathrm{dBc} / \mathrm{Hz}$ and decreases linearly until $10 \mathrm{kHz}$ where it reaches to $-116 \mathrm{dBc} / \mathrm{Hz}$. The first supermode noise spur is at $\sim 200 \mathrm{kHz}$ and suppressed $-75 \mathrm{~dB}$ from the carrier. As a comparison, the phase noise measurement of the commercial signal generator Keysight PSG E8257D with low phase noise option is also given.

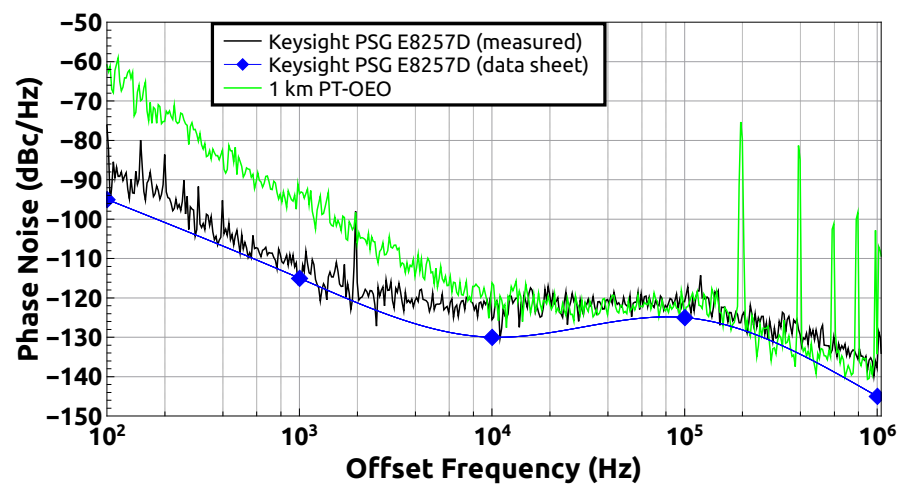

Fig. 3: Phase noise measurement of the all-fiber coupled $\mathscr{P} \mathscr{T}$-OEO setup and a commercial signal generator.

\section{Conclusion}

We have demonstrated an all-fiber coupled simple $\mathscr{P} \mathscr{T}$-OEO setup that can generate single-mode microwave signal at $9.5 \mathrm{GHz}$, without using an RF filter. We obtained a a phase noise value of $-116.2 \mathrm{dBc} / \mathrm{Hz}$ at $10 \mathrm{kHz}$ offset frequency. The next step planned is decreasing the phase noise of this setup by implementing longer delay lines and utilizing it in various optoelectronic systems.

This work is partially supported by the Turkish Academy of Sciences (TUBA) and the Scientific and Technological Research Council of Turkey (TUBITAK) under the grant 119F265.

\section{References}

1. X. S. Yao and L. Maleki, IEEE journal Quantum Electron. 32, 1141 (1996).

2. J. Tang, T. Hao, W. Li, D. Domenech, R. Baños, P. Muñoz, N. Zhu, J. Capmany, and M. Li, Opt. Express 26, 12257 (2018).

3. X. S. Yao and L. Maleki, IEEE J. Quantum Electron. 36, 79 (2000).

4. E. Shumakher and G. Eisenstein, IEEE Photonics Technol. Lett. 20, 1881 (2008).

5. I. Ozdur, D. Mandridis, N. Hoghooghi, and P. J. Delfyett, J. Light. Technol. 28, 3100 (2010).

6. C. M. Bender and S. Boettcher, Phys. Rev. Lett. 80, 5243 (1998).

7. J. Zhang and J. Yao, Sci. Adv. 4, eaar6782 (2018). 